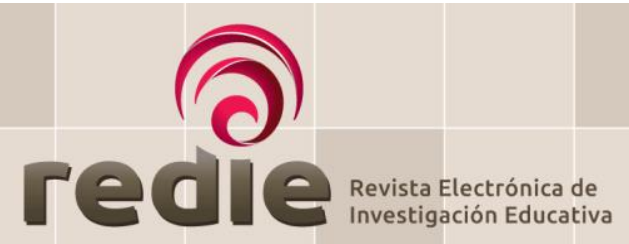

ISSN: $1607-4041$

https://redie.uabc.mx

Vol. 24, 2022/e02

\title{
Propiedades psicométricas del cuestionario sobre comportamiento lector y socialización familiar
}

\section{Psychometric Properties of a Questionnaire on Reading Behavior and Family Socialization}

\author{
Micaela Sánchez Martín (*) https://orcid.org/0000-0002-9107-574X \\ José David Cuesta Sáez de Tejeda (*) http://orcid.org/0000-0001-8500-0605 \\ Tomás Izquierdo Rus (*) https://orcid.org/0000-0003-0686-2747
}

(*) Universidad de Murcia, España

(Recibido: 2 de diciembre de 2019; Aceptado para su publicación: 13 de julio de 2020)

Cómo citar: Sánchez, M., Cuesta, J. D. e Izquierdo, T. (2022). Propiedades psicométricas del cuestionario sobre comportamiento lector y socialización familiar. Revista Electrónica de Investigación Educativa, 24, e02, 1-11.

https://doi.org/10.24320/redie.2022.24.e02.3648

\section{Resumen}

Son varios los factores implicados en el hábito lector de los estudiantes, siendo de los principales contextos de socialización lectora los ámbitos familiares y escolares. Este trabajo se centra en determinar las propiedades psicométricas de un cuestionario para identificar las principales variables que favorecen la adquisición del hábito lector en Educación Primaria, analizar el comportamiento lector en función de los libros leídos, y corroborar el modelo predictor del comportamiento lector con base en un modelo de regresión lineal. Desde un enfoque cuantitativo no experimental, de corte transversal, la validación del instrumento se realizó mediante el análisis de datos aportados por 403 estudiantes de Educación Primaria de la Región de Murcia (España), para lo cual se ha realizado la validez de constructo mediante el análisis factorial y la validez de consistencia y confiabilidad. Los resultados obtenidos indican que el instrumento construido posee unos indicadores psicométricos consistentes para ser utilizado con los fines que persigue.

Palabras clave: psicometría, hábito de lectura, enseñanza primaria, educación familiar

\begin{abstract}
A number of factors are associated with students' reading habit, and family and school environments are among the most important contexts for the socialization of reading. The focus of this study is to determine the psychometric properties of a questionnaire to identify the main variables that support the adoption of a reading habit in primary education, analyze reading behavior based on books read, and corroborate the predictive model for reading behavior using a linear regression model. Based on a non-experimental, crosssectional quantitative approach, the instrument was validated by analyzing data from 403 primary education students from the region of Murcia (Spain), and the construct validity was determined through factor analysis and by validating its consistency and reliability. The results obtained indicate that the instrument constructed has consistent psychometric indicators for the purpose it aims to serve.
\end{abstract}

Keywords: psychometrics, reading habit, primary education, family education 


\section{Introducción}

La lectura es un aspecto clave en el proceso educativo del alumnado y una de las mayores inquietudes de docentes, familias y estudiantes, ya que de su desarrollo en esta área dependerá el éxito o fracaso en el ámbito escolar. Además, entre el conjunto de representaciones que permite en diferentes ámbitos, se convierte en una herramienta de comunicación intrafamiliar (Bacilio, 2015), siendo necesaria la labor del profesorado para incentivar la implicación de las familias en el comportamiento lector (Martínez y Torres, 2019). Alcanzar un buen grado de comprensión lectora para un mejor desempeño académico y social pasa por la adquisición de un buen hábito de lectura por parte de los estudiantes. En este sentido, Molina (2006) subraya el valor de la lectura en todos los ámbitos sociales, fundamentalmente para la formación de hábitos lectores, y señala una relación directa entre el hábito lector de estudiantes de Educación Secundaria Obligatoria (ESO) y sus logros académicos. También la Ley Orgánica 87/2013 para la Mejora de la Calidad Educativa introduce como una de sus novedades el objetivo de fomentar el hábito y la adquisición de estrategias lectoescritoras, dedicando media hora al día de lectura y escritura en Primaria.

Aunque podemos encontrar algunos estudios que versan sobre el hábito lector, principalmente en Educación Secundaria (Çelik et al., 2015; Gil-Flores, 2009; Molina, 2006; Moreno, 2001, 2002), son más numerosos aquellos centrados en la comprensión lectora (Ülper et al., 2017), debido al auge que ésta ha tenido en los últimos años, por su presencia en las pruebas de evaluación y diagnóstico, tanto nacionales como internacionales. Entre las que cabe destacar el Estudio Internacional de Progreso en Comprensión Lectora (PIRLS) llevado a cabo por la Asociación Internacional para la Evaluación del Rendimiento Educativo (Ministerio de Educación, Cultura y Deporte, 2017), que evalúa la comprensión lectora entre el alumnado de 4o. de Educación Primaria, y cuyos resultados sitúan a España por debajo de la media de los países participantes de la Unión Europea y de la OCDE (Izquierdo et al., 2019). Datos que coinciden con los ofrecidos por la OCDE (2015) dentro del Programa para la Evaluación Internacional de Alumnos (PISA). O los aportados por el Barómetro de Hábitos de Lectura y Compra de Libros en España, publicado por la Federación de Gremios de Editores de España ([FGEE], 2018), cuyos resultados muestran por una parte que todos los niños de 10 a 13 años además de leer por motivos escolares leen con una frecuencia al menos trimestral en cualquier formato; y por otra, que por comunidades, la Región de Murcia es junto con Extremadura la que posee menor porcentaje de lectores de libros en el tiempo de ocio, con un 54.2\%, frente al 59.1\% de la media española.

Si bien un aspecto fundamental para el desarrollo de los hábitos lectores es la comprensión lectora, el gusto por leer y la motivación son actitudes valiosas para el aprendizaje de los estudiantes. La lectura no debe entenderse únicamente desde un punto de vista competencial o aplicado, como una destreza para entender y usar formas lingüísticas, sino también desde su dimensión como proceso constructivo e interactivo, en la medida en que sirve para construir significados y como finalidad enfocada al aprendizaje, al disfrute y la participación en la vida diaria (Ministerio de Educación, Cultura y Deporte, 2017). El hábito de lectura debe constituir una tarea que se consiga mediante el establecimiento de vocaciones y la animación permanente, favoreciendo situaciones de lectura creativas y recreativas (Molina, 2006), donde la lectura sea valorada y apreciada por encima de su valor meramente instrumental, considerándose su valor lúdico, como medio para el disfrute. Por lo que se convierte en necesario diferenciar entre la lectura realizada con fines de ocio y la lectura instrumental, impuesta por la escuela y asociada únicamente al ámbito académico.

El gusto por la lectura supone un aprendizaje por parte del niño que debe responder a sus intereses y motivaciones (Valdés, 2013). Sin embargo, desde el inicio del proceso educativo de los estudiantes la escuela impone la lectura, y los niños leen más por exigencias académicas que por propio interés. Según datos del Barómetro de Hábitos de Lectura y Compra de Libros en España (FGEE, 2018), los niños no sitúan la lectura entre sus actividades de ocio favoritas; es decir, no leen por voluntad propia ni manifiestan disfrute con la lectura. Como destaca Molina (2006) los tipos de ocio están cambiando, lo que se traduce en que actividades como la televisión, los videojuegos o internet están quitando tiempo para la lectura. Por ello, la lectura no ocupa un papel principal en el tiempo de ocio de los estudiantes, posicionándose por delante de la misma actividades como practicar deporte, escuchar música o estar con la familia (Muñoz y Hernández, 2011). Para que se puedan desarrollar los hábitos de lectura es necesario valorar y considerar que la lectura es culturalmente relevante, y no una mera destreza instrumental con carácter obligatorio, 
con el fin de evitar que quede relegada al espacio único de formación y que sea percibida por el alumnado como una obligación fuera del tiempo de ocio.

El análisis de las actividades que realizan las personas en su tiempo libre es clave para la evaluación de los comportamientos sociales. Así, al relacionar el tiempo que pasa el alumnado jugando videojuegos o viendo la televisión con la afición y el gusto por la lectura y el rendimiento académico, el rendimiento académico correlaciona de forma positiva con la lectura por placer, mientras que el uso inadecuado de videojuegos y televisión afecta las calificaciones académicas: a mayor cantidad de horas delante de la pantalla, peores resultados académicos (Dezcallar et al., 2014).

Por otra parte, las conductas del alumnado de Educación Primaria no se pueden atribuir únicamente a sus decisiones, sino que sus comportamientos se ven influenciados por los rasgos propios de su entorno próximo (Yubero y Larrañaga, 2010). De este modo el valor que los niños otorguen a la lectura dependerá de un conjunto de motivaciones y valores generados en su contexto social y cultural. Resulta difícil para un niño realizar acciones que las personas adultas de su entorno próximo no realizan; al ser la familia el principal contexto de desarrollo durante sus primeros años es de suma importancia su apoyo en la escuela para transmitir el gusto y el valor lúdico que tiene la lectura, ya que el papel que ésta ocupa en el desarrollo de sus hijos es crucial.

Las relaciones familiares, además de ser las primeras, son las más persistentes y se caracterizan por una especial intensidad afectiva y capacidad configuradora sobre las relaciones que tendrán lugar posteriormente fuera de la misma. Las actitudes familiares hacia la lectura inciden en el rendimiento académico en las asignaturas de lengua y matemáticas, y en los hábitos lectores, presentando mejores resultados académicos el alumnado cuyos padres dedican más tiempo y muestran una actitud más positiva hacia la lectura (Gil-Flores, 2009). En este contexto, la necesidad de apoyo por parte de las familias a la escuela para la educación de sus hijos se hace cada vez más evidente, y se materializa en aspectos como la comunicación con el profesorado del centro, el asesoramiento y la ayuda para realizar los deberes o el seguimiento del trabajo diario de sus hijos (Molero, 2003).

Teniendo en cuenta lo antes mencionado, en esta investigación construye un instrumento sobre el hábito lector y se analiza el comportamiento lector para corroborar el modelo de comportamiento lector del alumnado de 5o. y 6o. curso en centros de Educación Primaria de la Comunidad de Murcia, para ello se planteraron los siguientes objetivos:

- Determinar las características técnicas del cuestionario para identificar cuáles son las principales dimensiones implicadas en su estudio.

- Analizar el comportamiento lector en función de los libros leídos durante el curso y en vacaciones.

- Corroborar el modelo predictor del comportamiento lector.

\section{Método}

Con el fin de obtener una imagen lo más real posible de la situación actual sobre hábitos lectores entre los estudiantes de Educación Primaria en la Región de Murcia, se optó por una metodología cuantitativa no experimental de corte transversal, centrada en el diseño de un instrumento para identificar los principales factores que intervienen en el hábito lector de los estudiantes de Educación Primaria, que cumpla con los requisitos técnicos de validez y fiabilidad; así como la realización de un diseño predictivo transversal (DPT) para corroborar el comportamiento lector.

En el curso de la investigación se enviaron invitaciones a los centros para que participaran en la investigación. La recolección de los participantes tuvo lugar en junio de 2018. Se pidió a las familias interesadas en participar que se pusieran en contacto con el centro y complementaran un formulario de consentimiento. 
En la selección de la muestra se garantizó la presencia de colegios de zonas rurales y urbanas, de titularidad pública y privada, así como de contextos económicos y culturales heterogéneos: quedó compuesta por nueve colegios situados en Lorca, Águilas y Murcia. En este contexto diverso, se encontraron centros localizados en zonas con gran presencia de inmigrantes, y otros en zonas de alto nivel económico, aunque la mayoría se ubica en barrios de clase media. Es por ello, que, en esta pluralidad de alumnado, predomina el procedente de familias con un nivel económico y cultural medio. Cabe señalar que el índice de desventaja de la escuela es de tres sobre diez. Existen familias monoparentales y mixtas que viven en los municipios estudiados. Además, se pudo constatar que las madres son las que se ocupan en mayor medida de la escolarización de sus hijos, tanto por los comentarios emitidos por el profesorado como por el hecho de que a las reuniones informativas de la investigación se presentaron más las madres que los padres.

Como se observa en la Tabla 1, los participantes en esta investigación han sido 403 estudiantes de tercer ciclo de Educación Primaria, con una edad media de 11.05 (DT =1.09) de los cuales 200 son chicos y 203 chicas, seleccionados mediante un muestreo no probabilístico intencional.

Tabla 1. Características de los participantes

\begin{tabular}{llcc}
\hline & & $f$ & $\%$ \\
\hline \multirow{2}{*}{ Sexo } & Hombre & 200 & 49.6 \\
& Mujer & 203 & 50.4 \\
\hline \multirow{3}{*}{ Edad } & 10 años & 100 & 24.8 \\
& 11 años & 183 & 45.4 \\
& 12 años & 97 & 24.1 \\
\multirow{3}{*}{ Curso } & 13 años & 21 & 5.2 \\
\hline \multirow{2}{*}{ Titularidad del Centro } & Quinto & 194 & 48.1 \\
& Sexto & 209 & 51.9 \\
\hline
\end{tabular}

El uso de cuestionarios con fines educativos, encaminados a la mejora práctica docente, y con una considerable repercusión en el quehacer diario de las familias, debe basarse en el análisis la consistencia interna, la estabilidad y la capacidad de los resultados observados y los diferentes tipos de validez.

El instrumento utilizado para la recogida de información se construyó a partir del Cuestionario de Implicación de la Familia en la Educación Formal (Martínez-González,1995) adaptado por Molero (2003) y el Cuestionario sobre Lectura (Yubero y Larrañaga, 2010), quedando finalmente compuesto por:

- Preguntas de identificación: referidas a edad, género, tipo de centro, situación laboral y nivel económico y de estudios de los progenitores.

- El Cuestionario de Implicación de la Familia en la Educación Formal (Molero, 2003) mide la percepción que los estudiantes tienen sobre la implicación familiar en su proceso educativo. De los cuatro factores que componen este cuestionario se seleccionó el primer primero, correspondiente al interés-ayuda familiar. Éste consta de una escala tipo Likert con cinco niveles graduales de respuesta, que va desde nunca (1) hasta siempre (5), siendo .75 la fiabilidad alcanzada por los autores de la escala.

- El Cuestionario sobre lectura de Yubero y Larrañaga (2010) compuesto por preguntas referidas al comportamiento lector, valor de la lectura, motivación lectora, socialización parental y lugar que la lectura ocupa en sus actividades de ocio y tiempo libre. A la mayoría de estas cuestiones se responde mediante una escala Likert con cinco niveles graduales, que va desde nada (1) hasta mucho (5), siendo la fiabilidad alcanzada por los autores de la escala de valores de .81.

Tras la solicitud de los permisos oportunos y el establecimiento de la agenda se procede a la recogida de datos. Luego de garantizar el anonimato y confidencialidad de los datos, se procedió a la explicación del cuestionario y la resolución de dudas para su cumplimentación individual, se dieron 20 minutos para ello. 
Posteriormente los datos fueron analizados con el programa de análisis de datos cuantitativos SPSS Statistics 25.0 para ver el comportamiento y la estructura existente en dichos datos (Hernández et al., 2015). Con este fin se utilizaron técnicas de análisis factorial, cálculo de fiabilidad y análisis de regresión multivariante para corroborar el modelo predictor del comportamiento lector. El análisis factorial exploratorio se ha llevado a cabo mediante la reducción de factores. Para seleccionar el método de extracción, la rotación de la solución y el número de factores retenidos en la solución de factores se han tomado las siguientes decisiones:

a) Se verificó, mediante el coeficiente de asimetría y aplanamiento de la curva, que la distribución de los ítems es normal y justifica el uso del método de extracción de máxima probabilidad.

b) Los datos dicotómicos invitan a aplicar la rotación Varimax, que tiene como objetivo reducir al mínimo el número de elementos atribuidos a un factor determinado, maximizando la variación intrafactorial y promoviendo la diferencia entre las cargas de los factores altos y bajos. Esta rotación es apropiada tanto para escalas de tipo unidimensional como multidimensional. Por esta razón, es adecuado para el estudio del rasgo latente en las ciencias sociales, ya que acepta alguna correlación entre los factores.

c) El gráfico de sedimentación obtenido de los "autovalores" permite la identificación del número de factores que deben conservarse en la solución factorial.

\section{Resultados}

Los resultados para evaluar las características psicométricas se organizaron en varios apartados: estructura de factores y coherencia interna (análisis factorial y alfa de Cronbach). La consistencia interna fue comprobada calculando el lfa de Cronbach, que se esperaba que fuera superior a .7 pero menor a .95. La estructura de los factores fue comprobada luego por medio de análisis factorial para los datos ordinales, por el método de componentes principales y rotación Varimax.

\subsection{Estructura factorial}

Con la finalidad de determinar la validez de constructo se procedió a establecer las relaciones entre cada factor y los ítems que constituyen las variables del estudio, con objeto de establecer los factores que la componen. Se llevó a cabo el análisis factorial, utilizando el método de extracción de componentes principales, de los 27 ítems del cuestionario, los cuales responden a una escala con respuestas graduales en términos de frecuencia e importancia que determinan el tipo lector, a fin de delimitar los componentes principales y establecer la varianza total que éstos explican.

Рara comprobar la matriz de correlaciones se ha empleado el test de Bartlett (Tabla 2). Con este análisis obtenemos una matriz de identidad, la cual nos indica si todos los coeficientes de la diagonal son iguales a la unidad y los extremos de la diagonal iguales a cero. Este estadístico, obtenido desde las transformaciones de Chi-Cuadrado del determinante de la matriz de correlaciones, establece que cuanto menor sea su grado de significación y mayor su valor, aumenta la incertidumbre sobre la obtención de una matriz de identidad. El Chi-cuadrado obtenido arroja un valor de 3052.401 ( $p=.000)$, por lo que no se trata de una matriz de identidad, pudiendo corroborar que el análisis factorial resulta conveniente y que puede aportar conclusiones satisfactorias. Asimismo, la medida KMO indica una adecuación de .889, por lo que el resultado resulta conveniente para poder realizar el análisis factorial.

Tabla 2. Prueba de esferalidad de Bartlett

\begin{tabular}{lll}
\hline \multicolumn{2}{l}{ Medida Kaiser-Meyer-Olkin de adecuación de muestreo } & .889 \\
\hline Prueba de esfericidad de Bartlett & Aprox. Chi-cuadrado & 3052.401 \\
\hline & Gl & 253 \\
\hline
\end{tabular}


Para la extracción de factores y rotación se ha empleado el modelo el de Componentes Principales (MCP), en el que mediante la transformación de los datos iniciales en nuevos factores se obtiene una serie de componentes que explican la máxima varianza total de las variables iniciales. Se ha seleccionado esta técnica, con carácter exploratorio, por ser bastante aceptada en el ámbito de la investigación socioeducativa. Como se puede observar la Tabla 3, el instrumento se compone de cinco factores, con valores propios superiores a 1.

Tabla 3. Varianza total explicada de los cinco factores

\begin{tabular}{cccc|ccc}
\hline \multicolumn{3}{c}{} & \multicolumn{3}{c|}{ Autovalores iniciales } & \multicolumn{3}{c}{$\begin{array}{c}\text { Sumas de cargas al cuadrado } \\
\text { de la extracción }\end{array}$} \\
\hline Componente & Total & $\begin{array}{c}\text { \% de } \\
\text { varianza }\end{array}$ & $\begin{array}{c}\text { \% } \\
\text { acumulado }\end{array}$ & Total & $\begin{array}{c}\text { \% de } \\
\text { varianza }\end{array}$ & $\begin{array}{c}\text { \% acumulado } \\
\text { and }\end{array}$ \\
\hline 1 & 7.244 & 31.496 & 31.496 & 7.244 & 31.496 & 31.496 \\
2 & 1.799 & 7.823 & 39.319 & 1.799 & 7.823 & 39.319 \\
3 & 1.603 & 6.971 & 46.291 & 1.603 & 6.971 & 46.291 \\
4 & 1.213 & 5.275 & 51.565 & 1.213 & 5.275 & 51.565 \\
5 & 1.074 & 4.668 & 56.233 & 1.074 & 4.668 & 56.233 \\
\hline
\end{tabular}

La Tabla 4 presenta el resultado del análisis de los datos empleando el Método de Componentes Principales (MCP) y la rotación Varimax con Kaiser, para minimizar el número de variables con que presentan una saturación elevada en cada factor. En la que se puede observar los porcentajes individuales y acumulados; así como la proporción de varianza total explicada por cada factor, tanto para la solución no rotada como para la rotada.

Tabla 4. Matriz de componentes principales

\begin{tabular}{|c|c|c|c|c|c|}
\hline & 1 & 2 & 3 & 4 & 5 \\
\hline Leer un rato todos los días & .770 & & & & \\
\hline Cuidar los libros & & & & .575 & \\
\hline La lectura me importa & .745 & & & & \\
\hline Conocer y haber leído muchos libros & 690 & & & & \\
\hline Leer рага aprender & & & & .496 & \\
\hline Disfrutar parte de mi tiempo leyendo un libro & .738 & & & & \\
\hline Compartir con mis amigos las lecturas & & & & & .573 \\
\hline Ser lector te ayuda a ser mejor en el futuro & & & & .522 & \\
\hline Tener libros en casa & & & & .616 & \\
\hline ¿Te gusta leer? & .788 & & & & \\
\hline ¿Cuánto crees que le gusta la lectura a tu padre? & & .743 & & & \\
\hline ¿Cuánto crees que le gusta la lectura a tu madre? & & .707 & & & \\
\hline ¿Cuánto crees que le gusta la lectura a tu profesor/a? & & & & & .758 \\
\hline ¿Cuánto crees que le gusta la lectura a tu amigos/as? & & & & & .544 \\
\hline ¿Cuánto crees que le gusta la lectura a tu hermano/a? & & .425 & & & \\
\hline Leer un rato todos los días & .770 & & & & \\
\hline ¿Ves leer a tus padres durante su tiempo libre? & & .753 & & & \\
\hline ¿Cuántos libros has leído este curso? & & & .742 & & \\
\hline ¿Cuántos libros lees en vacaciones? & & & .749 & & \\
\hline ¿Tus padres te regalan libros? & & & .734 & & \\
\hline ¿Te recomiendan libros para leer? & .665 & & & & \\
\hline ¿Leéis juntos algún libro? & & .516 & & & \\
\hline ¿Comentáis libros de lectura? & & .465 & & & \\
\hline
\end{tabular}

Los factores hallados se han denominado a partir de las diferentes dimensiones que determinan el hábito lector de los estudiantes de tercer ciclo de Educación Primaria en los 403 cuestionarios analizados y su relación con los elementos que componen cada una de ellas. A los cinco factores obtenidos del modelo, que explican un 56.223\% de la varianza total, se les ha asignado la siguiente denominación: 
I. Interés por la lectura: factor compuesto por 6 ítems y explica un $31.496 \%$ de la varianza total.

II. Hábitos lectores familiares: constituido por 6 ítems, que explica un $7.823 \%$ de la varianza total.

III. Cantidad de libros leídos. Compuesto de 3 ítems, explica un $6.971 \%$ de la varianza total.

IV. Recursos, medios y utilidad de la lectura: constituido por 4 ítems, que explica el $5.275 \%$ de la varianza.

V. Preferencias lectoras en el entorno próximo: este último factor se compone de 3 ítems y explica el porcentaje menor de varianza, $4.668 \%$.

\subsection{Fiabilidad}

Con la finalidad de determinar las características de consistencia interna del cuestionario, se calculó la fiabilidad mediante el coeficiente a (alfa de Cronbach), obteniéndose un coeficiente global igual a .776, el coeficiente para el factor I (Interés por la lectura) es de .888, de .750 para el factor II (Hábitos lectores familiares), de .733, en el factor III (Cantidad de libros leídos), de .713, para el factor IV (Recursos, medios y utilidad de la lectura), y de .693 para el factor V (Preferencias lectoras en el entorno próximo).

\section{Comportamiento lector en función de los libros leídos durante el curso y en vacaciones}

Para analizar el comportamiento lector se ha establecido una tipología de lectores según la frecuencia con que se realiza la lectura, siguiendo las orientaciones de Yubero y Larrañaga (2010) y de los informes nacionales de la Federación de Gremios de Editores de España (FGEE, 2018). Para ello, se ha cruzado el número de libros leídos por el alumnado durante el curso con los leídos durante vacaciones, estableciendo tres categorías de lectores:

1) No lectores: alumnado que durante el curso lee sólo las lecturas obligatorias del colegio y no lee nada durante vacaciones.

2) Lectores ocasionales: estudiantes que leen uno o dos libros además de las lecturas impuestas por el colegio y algún libro durante las vacaciones (incluye a niños que leen mensual o trimestralmente).

3) Lectores frecuentes: niños que leen casi a diario o varias veces por semana (comprende a los estudiantes que durante el curso escolar han leído más de 5 libros y que, además, leen en vacaciones).

La Tabla 5 muestra que la mayor parte del alumnado se ubica dentro de la categoría de lectores frecuentes (49.6\%), seguidos por los lectores ocasionales (25.8\%) y los no lectores (24.6\%). 
Tabla 5. Comportamiento lector del alumnado de 2o. Ciclo de Educación Primaria (\%)

\begin{tabular}{|c|c|c|c|c|c|}
\hline \multirow{9}{*}{$\begin{array}{l}\text { Libros leídos } \\
\text { durante el curso }\end{array}$} & \multicolumn{5}{|c|}{ Libros leídos en vacaciones } \\
\hline & & Ninguno & 102 & 304 & Más de 5 \\
\hline & Ninguno & 0.74 & 0.49 & 0 & 0 \\
\hline & Uno & 2.72 & 1.48 & 0.74 & 0 \\
\hline & Dos & 1.48 & 2.72 & 0.49 & 0 \\
\hline & Tres & 1.98 & 3.72 & 2.23 & 0.24 \\
\hline & Cuatro & 1.24 & 8.18 & 3.72 & 2.23 \\
\hline & Cinco & 0.49 & 3.97 & 5.95 & 1.73 \\
\hline & Más de cinco & 3.72 & 12.15 & 21.33 & 16.12 \\
\hline
\end{tabular}

\section{Corroborar el modelo predictor del comportamiento lector}

Para corroborar el modelo predictor del comportamiento lector se ha efectuado un análisis de regresión lineal multivariante, arrojando los resultados una bondad de ajuste del modelo multivariante elevada con un $R^{2}$ corregido $=758$ y un error típico de la estimación bastante bajo $(p=.402)$, lo que indica que el modelo la adecuación del modelo para proceder a la estimación de los datos, y que las variables independientes incluidas en el conjunto (libros leídos en vacaciones y libros leídos durante el curso) contribuyen a explicar bastante bien la variable dependiente (tipo de lector). El estadístico $\mathrm{F}$ contrasta la significatividad global del modelo ( $F=421.368$ ), en el que se puede confirmar que probabilidad de que todos los coeficientes sean igual a cero es muy reducida $(p=.000)$, por lo que no se acepta la hipótesis nula de que todos los coeficientes sean igual a cero y el modelo se considera válido para predecir el tipo lector, pues como corroboran los resultados de la Tabla 6, el p-valor de las pruebas $t$ para cada una de las variables independientes es $\leq .05$ por lo que se rechaza la hipótesis nula. Siendo la variable independiente libros leídos en vacaciones la que presenta un mayor peso para explicar la variabilidad en la variable dependiente tipo de lector $(t=25.884, p \leq .05)$.

Tabla 6. Variables asociadas al tipo de lector para el modelo de regresión lineal en comportamiento lector

\begin{tabular}{lccccc}
\hline \multicolumn{1}{c}{ Modelo } & B & $\begin{array}{c}\text { Error } \\
\text { estándar }\end{array}$ & Beta & $t$ & p valor \\
\hline 1 (constante) & -.445 & .082 & & -5.401 & .000 \\
\hline Libros leidos curso & -.045 & .020 & -.066 & -2.232 & .026 \\
\hline Libros leídos vacaciones & .375 & .015 & .729 & 25.884 & .000 \\
\hline a. Variable dependiente: tipolector & & & &
\end{tabular}

\section{Discusión y conclusiones}

Respecto al primer objetivo, determinar las características técnicas del cuestionario, para identificar cuáles son las principales dimensiones implicadas en su estudio. El porcentaje de respuestas registradas en el estudio, basado en el análisis psicométrico, está en torno al 50\%, siendo ligeramente inferior a la observada en estudios similares (Ouellet et al., 2016; Sauvire, 2015), lo cual se debe a la naturaleza voluntaria y estrictamente anónima de la encuesta. Sin embargo, los resultados de trabajos análogos que registraron una tasa de respuesta de más del $80 \%$ no presentan una diferencia significativa con el promedio de otros estudios, resultados que permiten considerar que este estudio cumple con los siguientes criterios de confianza:

- Se construyó el Cuestionario para conocer la Implicación de la Socialización Familiar en el Comportamiento Lector del Alumnado de Educación Primaria (CISF-CLAEP), considerando los ya existentes (Molero, 2003; Yubero y Larrañaga, 2010), el cual ha demostrado tener buena consistencia interna y una validez de constructo satisfactoria. El análisis factorial exploratorio de componentes principales identificó una estructura de cinco factores que explican el $64.77 \%$ de la varianza total: Interés por la lectura, Hábitos lectores familiares, Cantidad de libros leídos, Recursos, medios y utilidad de la lectura y Preferencias lectoras en el entorno próximo. 
- La validez de constructo de la escala posee la suficiente estabilidad que se requiere para este tipo de instrumentos, ya que se apoya en las correlaciones significativas de Spearman, superando o igualando el umbral de .5 entre las respuestas, así como las correlaciones entre las puntuaciones medias en las diferentes subescalas y las otras variables.

Por lo que la validación de la escala ha permitido conocer sus características psicométricas y ofrece un instrumento válido y fiable para identificar el hábito lector del alumnado, así como la influencia de la familia, por lo que se trata de una herramienta útil para la práctica educativa que permite detectar e intervenir desde edades tempranas la adquisición y mejora del comportamiento lector de los niños, tanto desde el ámbito escolar como desde el familiar (Gil-Flores, 2009, Izquierdo et al., 2019; Moreno, 2001, 2002).

En lo relativo al segundo objetivo, analizar el comportamiento lector en función de los libros leídos durante el curso y en vacaciones, respecto a otros estudios (FGEE, 2018; Yubero y Larrañaga, 2010) se concluye que la cantidad de estudiantes lectores de los dos últimos cursos de Educación Primaria de los centros escolares de la Región de Murcia participantes en este estudio es levemente inferior a la media española, presentando mayores índices de lectura las chicas. Además, la Región de Murcia es junto con Extremadura la que posee menor porcentaje de lectores de libros en el tiempo ocio en España. Cifras que se mantienen a lo largo del tiempo a pesar de la importancia de crear un hábito lector entre nuestros estudiantes desde edades tempranas. De ahí la necesidad básica de programar acciones centradas en el fortalecimiento de la relación y la comunicación entre familia-escuela (Cárcamo y Garreta, 2020), para conseguir la adquisición de este hábito por parte de niñas y niños. Pues los comportamientos de los estudiantes de Educación Primaria se deben más a la influencia de los rasgos de su entorno próximo que a sus decisiones personales, por lo que el valor que los niños otorgan a la lectura depende principalmente del conjunto motivaciones y de valores generados en su contexto social y cultural (Izquierdo et al., 2019). En este sentido, profesorado, familia y alumnado han de realizar actividades orientadas a la mejora, el gusto y la afición por la lectura. Desde la escuela se han de promover de métodos novedosos mediante la creación de actividades lectoras atractivas y agradables, y desde sus hogares las familias deben cumplir con un rol parental de apoyo a las actividades desarrolladas en la escuela; ya que como señala Sánchez-Martín (2020) en estas se establecen las primeras relaciones, que se caracterizan por su capacidad configuradora de hábitos y su permanencia en el tiempo, que se manifestará posteriormente fuera de ella. Por lo que la familia tiene una responsabilidad ineludible en la adquisición del hábito lector.

Por otra parte, según Villalpando (2014) la perspectiva sociocultural considera bienes simbólicos tanto al libro como a la lectura y, ambos forman parte del capital cultural que transmiten principalmente los organismos, la familia y la escuela. Pero la lectura es percibida por los estudiantes, en primer lugar, como un medio instrumental con carácter obligatorio para aprender o mejorar sus trabajos escolares, y en segundo lugar como una actividad de entretenimiento. Para que se puedan desarrollar los hábitos de lectura es necesario valorar y considerar la lectura como culturalmente relevante, gozando de la actividad para que se convierta en un hábito.

En el último objetivo, Corroborar el modelo predictor del comportamiento lector, el modelo predictor del comportamiento lector propuesto por la FGEE (2018) y Yubero y Larrañaga (2010), queda ratificado mediante el análisis de regresión lineal multivariante, ya que se confirman las variables libros leídos en vacaciones y libros leídos durante el curso como predictoras sobre el tipo de lector. En este sentido Villalpando (2014), en un estudio realizado en Jalisco (México) sobre el consumo cultural y la lectura, destaca el peso del nivel sociocultural familiar, la edad de inicio a la lectura, así como de las personas que los motivan a leer; y detecta una correlación entre el período de escolaridad y la lectura entre los estudiantes de Educación Secundaria, por lo que la salida del sistema escolar supone la finalización de la práctica lectora para muchos de estos jóvenes. De ahí la importancia de la implicación familiar desde los primeros años en la animación a la lectura, especialmente durante los períodos vacacionales y tiempo de ocio, ya que leer durante vacaciones es la variable de mayor peso en el modelo predictor del comportamiento lector. Mostrando éste el modelo y, en consonancia con el objetivo anterior, la ineludible necesidad de estrechar lazos entre familia y escuela, por ser los dos principales agentes de socialización primaria, especialmente de los 0 a los 6 años, y responsables de la educación de los niños (Ramírez-García et al., 2018). 
Frente a las dificultades experimentadas por los estudiantes en la lectura, puede ser relevante para el profesorado que pueden practicar sobre cómo reflexionan, cómo perciben y cómo utilizan la lectura los estudiantes bajo el prisma de una herramienta pedagógica. Esta reflexión y la responsabilidad no sólo debe recaer en los profesores. La lectura concierne a todo el contexto que rodea al alumno, incluida la familia, su carácter transversal se incardina en la naturaleza de los textos leídos y según la experiencia y la pericia del lector.

\section{Referencias}

Bacilio, J. C. (2015). Representaciones sociales de la lectura en docentes de nivel primaria. Revista Iberoamericana para la Investigación y el Desarrollo Educativo, 6(11), 227-245.

https://www.ride.org.mx/index.php/RIDE/article/view/169

Cárcamo, H. y Garreta, J. (2020). Representaciones sociales de la relación familia-escuela desde la formación inicial del profesorado. Revista Electrónica de Investigación Educativa, 22(11), 1-14.

https://doi.org/10.24320/redie.2020.22.e11.2406

Çelik, T., Demirgüneş, S. y Fidan, D. (2015). Analysing the relation between reader-friendly texts reading comprehension competence. Başkent University Journal of Education, 2(1), 115-122.

http://buje.baskent.edu.tr/index.php/buje/article/view/29

Dezcallar, T., Clariana, M., Cladellas, R., Badia, M. y Gotzens, C. (2014). La lectura por placer: su incidencia en el rendimiento académico, las horas de televisión y las horas de videojuegos. OCNOS, (12), 107-116.

https://doi.org/10.18239/ocnos 2014.12.05

Federación de Gremios de Editores de España (FGEE). (2018). Barómetro de hábitos de lectura y compra de libros en España 2017. https://www.federacioneditores.org/lectura-y-compra-de-libros-2018.pdf

Gil-Flores, J. (2009). Hábitos y actitudes de las familias hacia la lectura y competencias básicas del alumnado. Revista de Educación, (350), 301-322. http://www.educacionyfp.gob.es/revista-de-educacion/numerosrevista-educacion/numeros-anteriores/2009/re350/re350-13.html

Hernández, F., Maquilón, J. J., Cuesta, J. D. e Izquierdo, T. (Eds.). (2015). Investigación y análisis de datos para la realización de TFG, TFM y tesis doctoral. Compobell.

Izquierdo, T., Sánchez, M. y López, M. D. (2019). Determinantes del entorno familiar en el fomento del hábito lector del alumnado de Educación Primaria. Estudios sobre Educación, 36, 157-179.

https://doi.org/10.15581/004.36.157-179

Ley Orgánica 8/2013, de 9 de diciembre, para la mejora de la calidad educativa. (2013). Boletín Oficial del Estado, 295, de 10 de diciembre de 2013, 97858- 97921. https://www.boe.es/diario boe/txt.php?id=BOEA-2013-12886

Martínez, M. M. y Torres, A. (2019). Hábito lector en estudiantes de primaria: influencia familiar y del plan lector del centro escolar. Revista Fuentes, 21(1), 103-114.

http://dx.doi.org/10.12795/revistafuentes.2018.v21.i1.07

Ministerio de Educación, Cultura y Deporte (2017). PIRLS-TIMS 2016. Estudio Internacional de progreso en comprensión lectora. IEA. Informe español. Secretaría General Técnica.

https://fracasoacademico.files.wordpress.com/2017/12/pirls-2016-informe-nacional.pdf

Molero, D. (2003). Estudio sobre la implicación de las familias en el desarrollo de los procesos de enseñanza y aprendizaje. Revista Española de Orientación y Pedagogía, 14(1), 61-82.

https://doi.org/10.5944/reop.vol.14.num.1.2003.11605 
Molina, L. (2006). Lectura y educación: los hábitos lectores y su repercusión académica en Educación Secundaria Obligatoria. OCNOS, (2), 105-122. https://doi.org/10.18239/ocnos 2006.02.07

Moreno, E. (2001). Análisis de la influencia de la familia en los hábitos lectores de sus hijas e hijos: un estudio etnográfico. Contextos Educativos, (4), 177-196.

https://publicaciones.unirioja.es/ojs/index.php/contextos/article/view/492

Moreno, E. (2002). La familia como institución sociocultural: su papel en la adquisición de los hábitos lectores. Portularia, (2), 309-324. http://hdl.handle.net/10272/130

Muñoz, J. M. y Hernández, A. (2011). Hábitos lectores de los alumnos de la ESO en la provincia de Salamanca. ¿Son el género y el entorno factores diferenciales? Revista de Educación, (354), 605-625.

http://dx.doi.org/ 10.4438/1988-592X-RE-2011-354-013

OCDE. (2015). PISA 2015. Programa para la evaluación internacional de los alumnos. Informe español. https://www.educacionyfp.gob.es/dctm/inee/internacional/pisa-

2015/pisa2015preliminarok.pdf?documentld=0901e72b8228b93c

Ouellet, C., Dubé, F. y Boultif, A. (2016). Mieux comprendre les textes disciplinaires: un défi au collégial [Comprender mejor los textos disciplinares: un desafío a nivel universitario]. Revue Correspondance, 9-12.

Ramírez-García, A., Anguita-López, V. y Casas, J. C. (2018). Escala de diagnóstico de las relaciones familiaescuela: análisis psicométrico. Revista Electrónica de Investigación Educativa, 20(2), 69-79.

https://doi.org/10.24320/redie.2018.20.2.1529

Sánchez-Martín, M. (2020). Influencias socio-familiares en la elección de los estudios de formación profesional. Revista Latinoamericana de Estudios de Familia, 12(1), 44-62.

http://revlatinofamilia.ucaldas.edu.co/downloads/Rlef12(1) 4.pdf

Sauvire, M. (2015). Le rôle des pairs dans l'interprétation du texte littéraire [El papel de los pares en la interpretación del texto literario]. Correspondance, 20(2), 7-10. https://n9.cl/uzvhu

Ülper, H., Çetinkaya, G. y Bayat, N. (2017). Okuduğunu anlama testinin geliştirilmesi [Elaboración de pruebas de comprensión lectora]. Ahi Evran University Journal of Kırşehir Education Faculty (KEFAD), 18(1), 175-187.

Valdés, M. (2013). ¿Leen de forma voluntaria y recreativa los niños que logran un buen nivel de comprensión lectora? OCNOS, (10), 71-89. https://www.redalyc.org/articulo.oa?id=259129174004

Villalpando, M. E. (2014). Consumo cultural del libro y la lectura en estudiantes de secundaria en Jalisco. Revista Electrónica de Investigación Educativa, 16(3), 54-70.

https://redie.uabc.mx/redie/article/view/539/958

Yubero, S. y Larrañaga, E. (2010). El valor de la lectura en relación con el comportamiento lector. Un estudio sobre los hábitos lectores y el estilo de vida en niños. OCNOS, (6), 7-20.

https://doi.org/10.18239/ocnos 2010.06.01 